\title{
Vibromyographic analysis of knee muscle imbalances in knee osteoarthritis
}

\author{
Kenneth McLeod, Sara Morley, Andrew Matsushima \\ Clinical Science and Engineering Research Center, Binghamton University, Binghamton, USA \\ Email: kmcleod@binghamton.edu
}

Received 2 February 2012; revised 27 February 2012; accepted 12 March 2012

\begin{abstract}
Muscle imbalances are a well accepted cause of musculo-skeletal pain, yet clinicians lack a simple, noninvasive, and reproducible means to assess muscle effort during activities of daily living. To address this issue, we have been developing vibromyography (VMG) as a means to quantitatively assess muscle mechanical effort such that accurate muscle effort ratios can be determined in the clinic. In this study, VMG was used to characterize muscle imbalances in older adults with low-level knee pain while they performed step-up and step-down activities. In addition to determining whether VMG can identify the imbalances previously reported in individuals with knee pain, we address the question of whether these knee muscle imbalances were a likely cause of knee pain, or whether the imbalances represented a coping response for pre-existing knee pain. A population ( $N=$ 30) of 35 - 85 year old men and women with a total of 42 painful knees were assessed. Robust multiple stepwise regression analysis identified weak hip abductor muscle effort relative to hamstring muscle effort as the best predictor of knee pain $(p=0.00006)$, with weak vastus lateralis effort in eccentric contraction, relative to concentric contraction, being the second best predictor $(p=\mathbf{0 . 0 0 0 3})$. Muscle imbalances were able to account for $40 \%$ of the variation in reported pain, and the observed pattern of increasing knee pain with increasing quadriceps strength during concentric contraction leads us to infer that the observed muscle imbalances are not the result of a protective action by the individual, but rather are a principle cause of the knee pain.
\end{abstract}

Keywords: Vibromyography; Knee Pain; Muscle Balance; Osteoarthritis

\section{INTRODUCTION}

Knee pain is the most commonly reported musculoskeletal pain condition and is associated with significant disability and remarkably poor quality of life [1]. Health surveys indicate that over $25 \%$ of adults report a recent occurrence of knee pain [2], while almost $40 \%$ of older adults report current knee pain, resulting in over 10 million (non-injury related) ambulatory medical care visits each year in the U.S. Moreover, the prevalence rates of knee pain are showing dramatic secular increases [3]. Interestingly, only two-thirds of individuals with radiographic evidence of knee osteoarthritis (OA) report knee pain, while no radiographic evidence of joint damage is seen in $75 \%$ of individuals reporting any degree of knee pain [4]. This observation has led to the suggestion that improper use of the joint, or knee muscle imbalance, is likely the primary cause of knee pain $[5,6]$. Correspondingly, if the muscle imbalance could be identified, then correction of the imbalance through focused exercise and/or behavioral modifications has the potential to both eliminate the pain, and reduce the long term risk of joint tissue injury in most individuals [7]. This clinical strategy has recently received the support of the clinical community [8].

Numerous muscle imbalances have previously been shown to be associated with knee pain. For example, anterior knee pain experienced during stair climbing, prolonged sitting, and kneeling is emblematic of patella femoral pain syndrome (PFPS), a condition generally believed to arise from the abnormal tracking of the patella due to weakness in the vastus medialis relative to the vastus lateralis [9]. Recent work also indicates that not only peak muscle effort, but also the activation timing of the two muscles may play a role in the etiology of PFPS [10]. Quadriceps weakness has been reported in numerous studies to precede the development of OA and to be a more important determinant of pain and disability than radiologic changes to bone and cartilage [11]. Recent EMG based studies have identified unusually high co-activation levels of the biceps femoris during activities of daily living, in particular during stair ascent, when the quadriceps are operating at close to $100 \%$ maximal concentric contraction [12]. In addition, weakness in hip abductors has been proposed as an explanation for why 
the medial tibio-femoral compartment is most often affected in knee OA. Weak stance limb abductors permit a drop of the pelvis towards the contralateral limb, resulting in increased loading on the medial compartment of the stance leg [13]. Correspondingly, completion of an eight week regimen of hip abductor exercises in a population of individuals with knee $\mathrm{OA}$ has been shown to result in a significant reduction of pain on the WOMAC pain scale [14].

Application of this knowledge of muscle imbalance, however, has been limited as neither EMG nor dynamometry readily lend themselves to assessment of multiple muscles during dynamic functional activity in the clinic. As an alternative to EMG or dynamometry, studies beginning in the 1990s have indicated that muscle effort assessment by vibromyography (VMG), may provide a more accurate, and more readily obtainable, means to identify critical muscle imbalances [15-18]. VMG recording techniques directly assess mechanical activity of individual muscles, and can be obtained utilizing a single sensor, features which reduce many of the complications which arise in obtaining and interpreting EMG or dynamometer measurements. While VMG recordings can be influenced by other confounding influences, such as bulk muscle motion and physiologic tremor [19], signal processing techniques such as wavelet packet analysis permits isolation of the VMG components associated with specific muscle fiber type contractions, thereby allowing an assessment of muscle activity through the full range of voluntary muscle contraction [20,21]. We have recently developed these techniques to permit the analysis of superficial muscle bodies which are predominantly composed of Type II muscle fibers [22].

The majority of studies on muscle imbalance in knee OA have involved comparisons between healthy and affected populations, however, in such studies it is difficult to determine whether the observed imbalances represent a coping strategy directed towards reducing pain during functional activity or whether the imbalance is an a-priori mal-adaptation. To address this question, we elected to investigate knee muscle imbalance in individuals with reported knee pain. We hypothesized that if the pain was a reflection of mal-adaptation, then increased imbalance should be observed which would exacerbate pain level. Conversely, if the imbalance arose as a coping mechanism, the imbalances observed should be consistent with protecting the knee from further injury as knee pain level increases. To address this hypothesis, we utilized VMG recordings to simultaneously assess knee muscle effort during both concentric and eccentric contraction in those muscles where imbalances have been previously implicated as contributing to knee pain, i.e., the quadriceps, hamstrings, and hip abductors.

\section{METHODS}

\subsection{Subjects}

The study protocol was approved by the Binghamton University Institutional Review Board. Adult subjects (men and women) over age 35 years who were currently experiencing, or had experienced within the past year, knee pain, were recruited from the University community, local community centers, as well as senior living centers. After obtaining informed consent, self-reported weight and height, exercise history, along with an estimation of knee pain level on a $10 \mathrm{~cm}$ visual analog scale (VAS) was obtained from each individual. If bilateral knee pain was reported, a VAS pain score was obtained for each knee individually. Exclusion criteria included a clinical diagnosis (radiographic evidence) of osteoarthritis, or recent knee surgery. Individuals who were taking medication for knee pain, or anyone who reported that they participated in "intense" exercise on a regular basis, that is, athletes whose knee muscle use pattern was strongly influenced by activities other than those of daily living, were also excluded.

\subsection{Muscle Effort Measurements}

Muscle effort was assessed using a 6-channel vibromyography (VMG) recording apparatus built to our technical specifications (SUNY Center for Advanced Sensor Technology, Stony Brook, NY). Muscle effort of the vastus lateralis (VL), vastus medialis (VM), biceps femoris (BF) and Sartorius (Sr) were obtained simultaneously using four transducers, each approximately $3 \mathrm{~cm}$ in diameter. The VMG transducer for the VL was positioned approximately $8 \mathrm{~cm}$ superior to the patella, and $8 \mathrm{~cm}$ lateral to the anterior midline of the thigh; the VM transducer was positioned approximately $5 \mathrm{~cm}$ superior to the patella and $5 \mathrm{~cm}$ medial of the thigh anterior midline; the BF transducer was positioned mid-thigh, approximately $3 \mathrm{~cm}$ lateral of the posterior midline of the thigh; and, the Sr transducer was positioned on the medial aspect of the thigh, halfway between the crotch and superior aspect of the patella. As the Sartorius muscle can be difficult to locate, test runs were completed with the subject performing a retiré or passé movement, thereby activating the sartorius (abducting the hip while flexing the knee), to ensure the $\mathrm{Sr}$ transducer was not picking up VM activity. Transducers were secured in place using a $2 \mathrm{~cm}$ elastic strap around the thigh, which also served to maintain a radial pressure of approximately $10,000 \mathrm{~N} / \mathrm{m}^{2}$ against the muscle body.

\subsection{Procedure}

Subjects were asked to step-up onto a $40 \mathrm{~cm} \times 40 \mathrm{~cm}$ step with a height of approximately $2 / 3$ the length of 
their tibia. For most subjects this corresponded to a 25 $\mathrm{cm}$ step, though several of the shorter individuals utilized a $20 \mathrm{~cm}$ step. The recording procedure began with the foot of the recording leg resting on the step. At the start of recording the individual rose to a single leg stance, paused, then returned to a resting position on the contralateral leg. Following a three second rest period, the procedure was repeated for a total of ten repetitions. Following completion, if pain was also reported in the contralateral knee, the process was repeated on the alternate leg. The set-up and recording process typically required 15 - 20 minutes for a single leg assessment.

\subsection{Data Analysis}

Muscle vibration signals obtained from the VMG transducers were processed into muscle effort via custom software which performs a time-frequency analysis on data streams collected at a $2 \mathrm{KHz}$ sampling rate with 16 bit resolution. In brief, spectral components below $30 \mathrm{~Hz}$ and above $250 \mathrm{~Hz}$ were removed by analog filtering in the transducer, after which the VMG signal underwent wavelet packet decomposition (Daubechies $8^{\text {th }}$ order) which allows isolation of time-frequency components associated predominantly with Type II muscle fiber activity (central frequencies of $70-90 \mathrm{~Hz}$ ), after which the amplitude envelop of the decomposed signal is obtained, which was then low pass filtered at $3 \mathrm{~Hz}$, allowing temporal resolution of less than $100 \mathrm{~ms}$. The average muscle effort for a repeated event was obtained by eliminating the first and last events, identifying the center time point of the remaining eight events, aligning the events at this center time point, and obtaining an overall event average.

Maximum muscle effort for these averaged events was calculated by integrating the resulting averaged muscle effort signal over a period defined between where the VL effort first reached $50 \%$ of peak value, to a point where VL returned to below $50 \%$ of peak value, then dividing by this time period, that is, all muscle effort values were keyed to the activity of the VL. This analysis was undertaken for both step-up and step-down phases of the procedure. Respective muscle ratios were calculated directly from these maximum effort values.

\subsection{VMG Linearity}

We anticipated that some of the elderly individuals may require close to maximum voluntary VL muscle effort to accomplish the step-up procedure, and so evaluated the ability of the VMG technology to accurately report muscle effort at maximal isokinetic efforts. VMG response linearity was evaluated on the VL using dynamometer measurements of knee torque (Cybex System 3 Pro, Cybex, Medway, MA). Torque measurements were obtained under isokinetic contraction conditions so that the con- tribution of inertial loading forces would be minimized. A rate of $60^{\circ} / \mathrm{sec}$ was used, consistent with the knee angle rotation rates expected during the step-up procedure. Test subjects executed six knee extensions at maximum voluntary contraction levels through a $90^{\circ}$ range of motion $\left(90^{\circ}-0^{\circ}\right)$. Torque and VMG recordings were averaged for each contraction event during the time period during which knee angular velocity was constant $\left(\sim 75^{\circ}\right.$ $15^{\circ}$ ). Tibial length was obtained to permit correction for gravitational loading effects. Least squares regression analysis was utilized to obtain the relationship between total knee torque generated and the muscle effort levels determined through VMG analysis.

\subsection{Statistical Analysis}

All data were analyzed using SPlus version 8 (TIBCO Spotfire, Somerville, MA). Stepwise regression analysis was used to identify the specific muscle ratios most predictive of self-reported pain levels (response variable). To reduce the potential influence of outliers and/or heteroscedastic errors in this relatively small data set, MMRobust regression was utilized in all analyses. Given the large number of parameters involved in the analysis, a minimum p-value of 0.01 was used to establish significance.

\section{RESULTS}

Linearity testing on young men demonstrated that at maximum isokinetic $\left(60^{\circ} / \mathrm{sec}\right)$ voluntary contraction levels, knee extensions could be maintained for approximately $60^{\circ}$ of rotation $\left(\sim 75^{\circ}-15^{\circ}\right)$. Maximum torques achieved at this testing velocity ranged from about 100 $250 \mathrm{~N}-\mathrm{m}$, with peak torque achieved near a $60^{\circ}$ knee angle (Figure 1(a)). At these torque levels, correction for gravitational loading on the leg had minimal influence on total torque generated by the quadriceps. In the absence of any kinematic corrections, or for the contributions of multiple quadriceps muscles to knee extension, regression analyses of muscle effort, as reported by VMG, to measured torque resulted in regression coefficients $\left(R^{2}\right)$ greater than 0.7 (Figure 1(b)).

Thirty subjects who met the inclusion criteria were recruited and completed the study ( 8 men, 22 women). The average age of the subjects was 71 years (range $38-86$ years); their average body mass was $75 \mathrm{~kg}$ (range 48 $114 \mathrm{Kg}$ ); and they had an average BMI of $27 \mathrm{Kg} / \mathrm{m}^{2}$ (range $20-40 \mathrm{Kg} / \mathrm{m}^{2}$ ) (Table 1). Among these 30 subjects, 42 painful knees were reported. On a $0-10$ VAS scale, the maximum pain level reported was a 6 , and the average reported knee pain level was 3.2. The vast majority of volunteers were 60 years of age or older, and only one male subject was under age 65 years, however, no age dependence on reported pain levels was evident 
(Figure 2). Given the low fraction of men in the study, in all subsequent analyses, data from all subjects were ana-

Table 1. Demographic data on study population.

\begin{tabular}{cccc}
\hline Parameter & Mean & S.D. & N \\
\hline Age (years) & 71.1 & 11.4 & 30 \\
Weight (Kg) & 74.0 & 16.0 & 30 \\
Height (m) & 1.66 & 0.11 & 30 \\
BMI (Kg/m $\left.\mathbf{m}^{2}\right)$ & 27.1 & 4.4 & 30 \\
Self-reported knee pain & 3.2 & 1.6 & 42 \\
\hline
\end{tabular}

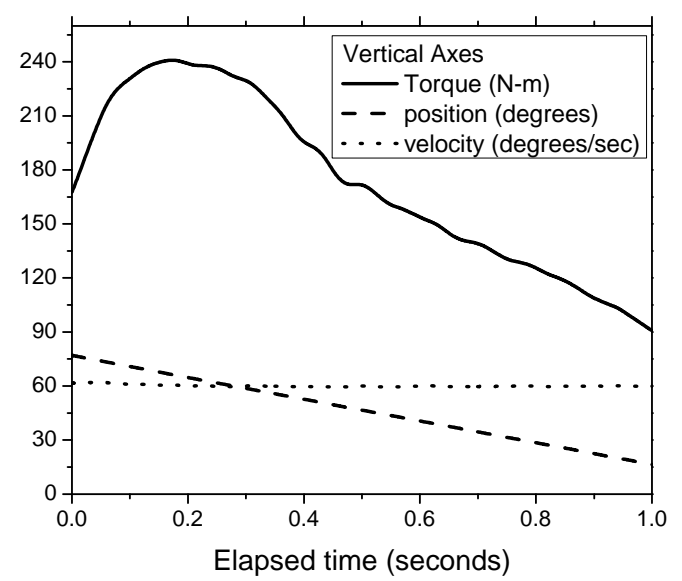

(a)

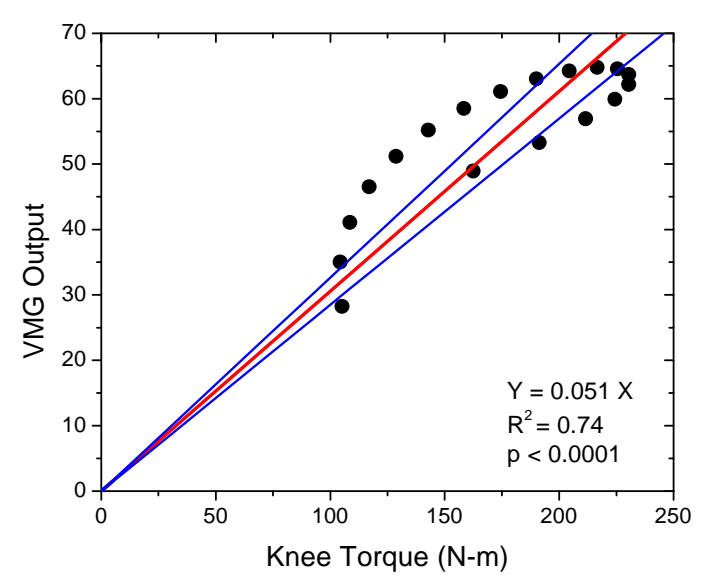

(b)

Figure 1. VMG response to maximum voluntary contraction during a $90^{\circ}$ knee extension. (a) Knee torque, angular velocity and angle of rotation during isokinetic contraction. Subjects were able to maintain $60 \%$ sec angular velocities for approximately $60^{\circ}$ of rotation. Peak torque is observed at knee angles of approximately $70^{\circ}-60^{\circ}$; (b) Muscle effort as obtained by VMG vs. torque, including correction for gravitational loading on the lower leg. A correlation coefficient of 0.75 is observed over the torque range of $100-250 \mathrm{~N}-\mathrm{m}(95 \%$ confidence limits on regression are shown).

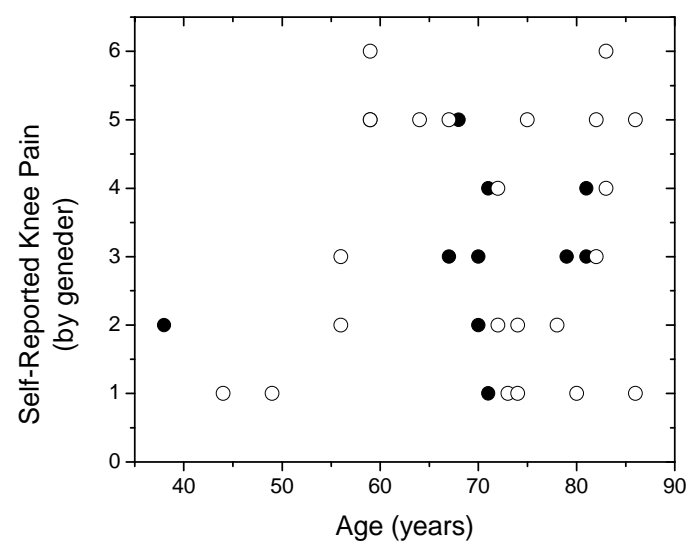

Figure 2. Self-reported knee pain levels for the study population (solid circles: men; open circles: women). Reported pain levels ranged from $1-6$ on a 10 point scale. Volunteers were predominantly over age 55 , and approximately $75 \%$ were women. Nationally, about $2 / 3$ of older individuals reporting knee pain are women. Age was not a significant predictor of pain level in this population.

lyzed as a pooled group.

The typical time for an individual to complete each step event in the protocol was about 4 seconds (not including the three second pause between events), with the time to complete the step-up and step-down phases being 1 - 1.5 seconds, and a 0.5 second one-legged stance phase (Figure 3). The muscle use pattern was similar in the majority of subjects. Maximal muscle effort in all four muscles typically peaked approximately half-way through the step-up and step-down phases. During the single legged stance phase, $\mathrm{BF}$ and $\mathrm{Sr}$ effort returned to close to resting levels, while VL and VM effort decreased substantially but typically remained well above resting level. VL and VM generally tracked each other closely throughout the events, and similarly, BF and $\mathrm{Sr}$ generally tracked each other closely, though at a level of effort which was typically one-half to one-third that of VL and VM.

Step-wise robust MM regression identified five parameters which were interpreted as significant predictors of self-reported knee pain level (Table 2). The most significant predictor $(\mathrm{p}=0.00006)$ was the ratio of the biceps femoris to sartorius muscle effort $(\mathrm{BF} / \mathrm{Sr})$ when stepping down, with higher $\mathrm{BF} / \mathrm{Sr}$ ratios being associated with higher pain levels. Interestingly, $\mathrm{BF} / \mathrm{Sr}$ during the step-up phase was also found to be a significant predictor of knee pain $(p=0.001)$, however, for the step-up phase of the event, higher $\mathrm{BF} / \mathrm{Sr}$ ratios were associated with lower knee pain levels. VL effort during the step-up phase (concentric contraction) relative to VL effort during the step-down phase (eccentric contraction) was found to be the second most significant predictor of knee pain $(p=0.0003)$. In individuals with minimal knee pain, 
Table 2. Regression analysis on knee pain as a function of measured muscle ratios and body mass. $\mathrm{N}=42$ knees.

\begin{tabular}{cccc}
\hline Parameter & Value & S.E. & t-value \\
\hline BF/Sr (Step-down) & 3.31 & 0.73 & 4.53 \\
$\mathbf{V L}_{\text {con }}$ /VL $_{\text {ecc }}$ & 1.45 & 0.37 & 3.94 \\
BF/Sr (Step-up) & -2.43 & 0.69 & -3.51 \\
VL/VM & 1.91 & 0.68 & 2.80 \\
Weight & 0.039 & 0.013 & 2.77 \\
\hline
\end{tabular}

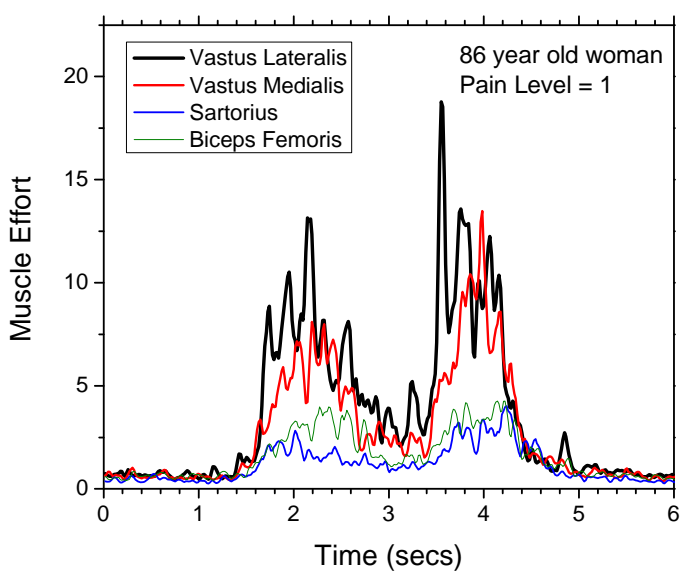

Figure 3. Typical muscle use pattern during the experimental procedure as assessed using VMG. Peak muscle force was generated by all four recorded muscles (VL, $\mathrm{VM}, \mathrm{BF}, \mathrm{Sr}$ ) approximately halfway through the step-up and the step-down phases. VL and VM were found to track each other closely, as well as BF and $\mathrm{Sr}$, however, $\mathrm{BF}$ and $\mathrm{Sr}$ effort levels were typically one-half to onethird that of the quadriceps muscles.

VL effort was seen to be substantially lower (by a factor of two) during step-up than during step-down (Figure 4), whereas those reporting a high level of knee pain demonstrated much higher VL activity during step-up than step-down. The VL/VM ratio was also observed to be a borderline significant predictor of knee pain, though only when the quadriceps were undergoing concentric contraction $(\mathrm{p}=0.008)$. Lastly, the weakest predictor identified in the multiple step-wise regression was body mass $(p=0.009)$. The combination of the above identified muscle effort ratios along with body mass was sufficient to explain $40 \%$ of the variation in reported pain levels.

\section{DISCUSSION}

In this study we investigated the ability of VMG recording techniques to provide estimates of muscle effort during functional activity as a clinical means to identify muscle imbalances in individuals with low level knee pain. Our results provide confirming evidence for the importance of several muscle imbalances which have

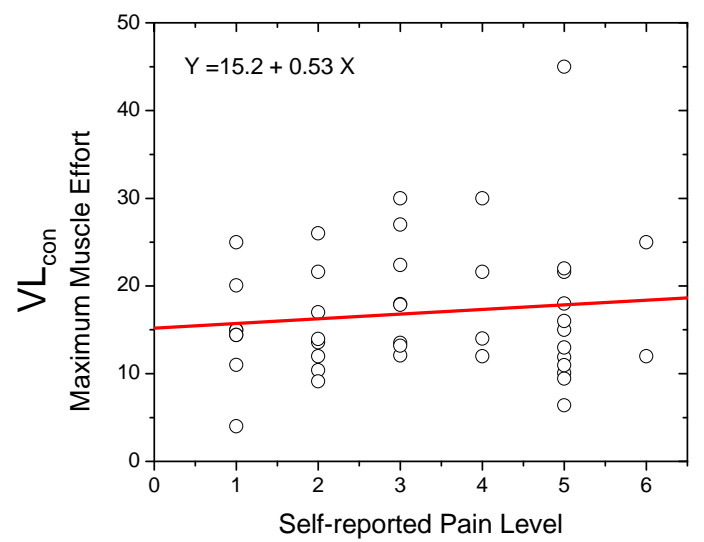

(a)

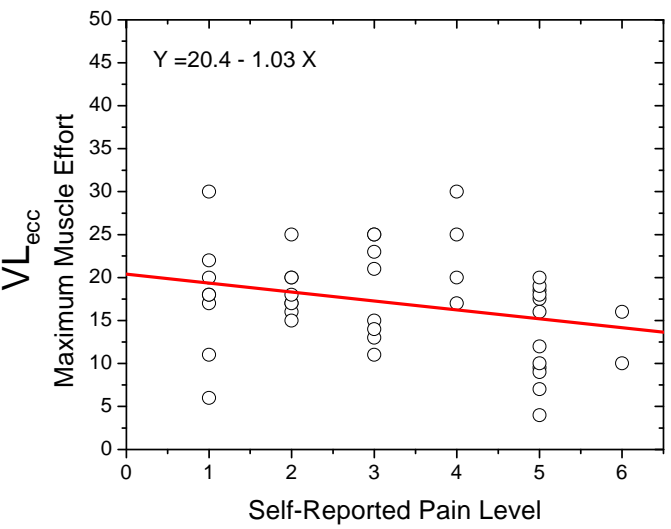

(b)

Figure 4. Vastus lateralis effort as a function of reported knee pain level. (a) Concentric contraction; (b) Eccentric contraction. No significant effects of pain on VL effort during step-up and step-down activity were observed in this population.

previously been reported as contributing to knee pain, but we were also able to identify important muscle balance ratios which, to our knowledge, have not previously been reported in the context of knee pain.

\subsection{VMG Performance}

We evaluated the ability of VMG to track maximal muscle effort under $60^{\circ} / \mathrm{sec}$ isokinetic contraction conditions and concluded that $R^{2}$ values in the range of 0.75 were sufficient to justify use of VMG in this study. As most of the subjects completed the step-up phase in about 1-1 1/2 seconds, and the $20-25 \mathrm{~cm}$ step typically requires a range of motion of approximately $60^{\circ}-80^{\circ}$, the $60^{\circ} / \mathrm{s}$ testing conditions seems to have been appropriate. Quadriceps muscles generate their maximum force near $60^{\circ}$ of flexion, and correspondingly, are less efficient at shorter or longer muscle lengths. Nonetheless, VMG appears to be capable of tracking the muscle force throughout this wide variation in muscle length. That the correlations between torque and VMG output were not higher may be 
the result of two factors not incorporated into these tests. Specifically, multiple quadriceps muscles contribute to knee extension, yet only the VL was evaluated here, though this was the muscle which we expected to come closest to being utilized at maximum dynamic contraction conditions given the study protocol. Inclusion of additional quadriceps muscles in the analysis would be straightforward from a recording perspective, but would require the development of an algorithm for determining how the individual muscle contributions should be combined to obtain an estimate of knee torque. In addition, the measured torque values were not converted to an estimate of muscle force by incorporating the kinematics of the knee joint. While the torque to muscle force relation is relatively flat in the region we analyzed $\left(15^{\circ}-75^{\circ}\right)$ [23], such correction may serve to substantially improve the correlations.

\subsection{Hamstring Hip-Abductor Ratios}

One distinct advantage of VMG is that it can provide an absolute measure of muscle effort, thereby allowing direct comparison of the effort in complementary muscle pairs. Among the four muscles we were evaluating in this study, the observed tracking of VL and VM was expected, but the remarkably close tracking of BF with $\mathrm{Sr}$ was an unexpected observation. Being a relatively weak hip abductor the Sartorius is not commonly studied, however, from the perspective of VMG recording the Sartorius is a particularly convenient hip abductor to record from as it is readily accessible on the medial side of the mid-thigh. While the role of hip abductors stabilizing the pelvis to prevent medial compartment loading has previously been discussed, it was interesting to find that the BF to $\mathrm{Sr}$ ratio (during step-down) was the strongest predictor of knee pain in our study population. This would seem to indicate that the $\mathrm{Sr}$ is a good surrogate abductor to evaluate in the clinic, though further studies doing direct comparisons between the Sartorius and other hip abductors should be pursued.

We were also intrigued that the $\mathrm{BF} / \mathrm{Sr}$ ratio during step-up was a significant predictor of knee pain, though in the reverse direction, that is, a higher $\mathrm{BF} / \mathrm{Sr}$ ratio during step-up was associated with less pain. This observation would appear to be consistent with the co-contraction role of the hamstrings in opposing the forward motion of the tibia during quadriceps contraction, however, in no situation did we find the quadriceps/hamstring ratio to be a significant predictor of knee pain. This observation, therefore, needs further investigation. In addition, the fact that $\mathrm{BF}$ and $\mathrm{Sr}$ muscle efforts are consistently one-half to one-third those observed in the VL and VM is consistent with their much smaller size, though this observation also seems to deserve further investigation given the importance of their roles in maintaining muscle balance during knee motion.

\subsection{VL-VM Ratio}

Consistent with numerous reports, we found the VL/VM ratio to be a significant predictor of knee pain, suggesting that PFPS was a significant contributor to the knee pain experienced by this population. While PFPS is a condition commonly associated with young people who regularly participate in repetitive knee motion activities (running, cycling, swimming, etc.), PFPS is also associated with extended sitting, kneeling and repeated stair climbing, activities that are likely to be a significant aspect of daily living for our older study population. Here, we addressed the VL/VM ratio only from an amplitude perspective, though timing was implicitly incorporated as a result of our cueing the analysis off the rise and fall times of the VL muscle during step-up and step-down. Because the VL/VM ratio during step-up turned out to be a significant predictor of pain and, it would likely be worthwhile to reevaluate the data looking explicitly for VM firing delays during step-down to see if this may be a more robust predictor of knee pain.

A particularly interesting observation from our data set is the strong relationship between knee pain and the ratio of peak VL force during step-up to that generated during step-down, that is, the respective VL forces generated during concentric vs. eccentric contraction. This ratio was the second most significant factor identified in our analysis, and shows a remarkable range, from approximately 0.5 , for those with minimal knee pain, to greater than 1.5 for those with high levels of knee pain. These results appear to be distinctly different from those reported by Hortobagyi, et al. [12]. In this latter EMG study involving both stair climbing and stair descent, the $\mathrm{VL}_{\text {con }} / \mathrm{VL}_{\mathrm{ecc}}$ ratios were found to be, on average, remarkably similar between individuals with OA (1.4), older individuals with no knee pain (1.1), and healthy young individuals (1.6). However, such EMG results may arise due to the well know fact that EMG signals increase under conditions of muscle fatigue, and so EMG results can be difficult to interpret when subjects are challenged to perform activities requiring maximum muscle effort. While the importance of eccentric quadriceps training has been reported for the treatment of jumper's knee [24], our data would seem to indicate that similar exercises may prove to be useful in the management of knee OA as well.

The $\mathrm{VL}_{\text {con }} / \mathrm{VL}_{\text {ecc }}$ data can also be utilized to address the main hypothesis of this study, that is, whether the observed imbalances reflect a coping mechanism for reducing pain of motion, or whether the pain has arisen as a result of a maladapted muscle use pattern. In princi- 
ple, such a determination would require a prospective study, however, our dose response data allows speculation on the consistency of the relationships between pain and the muscle forces being generated. Specifically, while we have shown that the $\mathrm{VL}_{\text {con }} / \mathrm{VL}_{\text {ecc }}$ ratio is strongly associated with knee pain levels, it is evident in Figure 4 that neither concentric VL strength nor eccentric VL strength demonstrate any significant trend at increasing pain levels. Therefore, the significant increase in the $\mathrm{VL}_{\mathrm{con}} / \mathrm{VL}_{\text {ecc }}$ ratio which is observed appears to be arising from concomitant small increases in concentric strength and corresponding decreases in eccentric strength. This is a rather natural development as many, if not most, activities of daily living rely much more on concentric quadriceps activity than on eccentric quadriceps activity. We therefore suggest that our data is consistent with the observed imbalances representing a slow drift in strength among the various muscle groups, at least in part due to body weight gain, resulting in significant muscle force imbalances which then give rise to inappropriate knee loading patterns which results in a painful knee. That is, we suggest that our data lends support to the hypothesis that knee pain follows the development of muscle imbalances, correspondingly, the correction of the observed imbalances would be expected to alleviate the knee pain reported by this study population.

\subsection{Limitations to the Study}

As a correlation analysis, this study cannot, of course, identify the causal basis of knee pain; such a conclusion would require follow-up to specific muscle training interventions. The main goal, therefore, was to determine whether VMG provided results consistent with previous reports which have relied on dynamometry and/or EMG analysis, such that an appropriate intervention study could be undertaken. This raises the issue of the accuracy and reproducibility of the observed results. Previous work has shown that VMG recording reproducibility (intra-assay variability) is $10 \%-15 \%$ for the muscles utilized in this study; nonetheless, with only 42 painful knees available for analysis, a multiple regression approach presents significant challenges. To minimize the risk of over interpreting the data set, we incorporated three quality control procedures; first, we utilized a stepwise regression approach, second, we utilized robust M-M regression to preclude spurious correlations, and finally, we set our significance value at a relatively high level ( $p$ $=0.01)$. Three of our observed correlations were well below this threshold, though two (VL/VM ratio and body mass) were of borderline statistical significance. A much larger study, in particular, one which included a larger range of pain levels, would be necessary to clarify the roles of these various muscle imbalances in the development of knee OA.

\section{SUMMARY AND CONCLUSIONS}

VMG is a relatively quick, and accurate, means to assess muscle effort and identify muscle force imbalances during dynamic functional activities involving both concentric and eccentric muscle contractions. VMG analysis of individuals with relatively low level knee pain $(\leq 6$ on a 10 point scale) has allowed isolation of several knee muscle imbalances associated with self-reported pain, in particular, the biceps femoris to Sartorius ratio, the vastus lateralis to vastus medialis ratio, and the vastus lateralis force ratio in concentric vs. eccentric contraction. These muscle imbalances, when combined with body weight, were found to be sufficient to explain $40 \%$ of the variation in the self-reported pain levels in a population of 35 - 85 year old men and women.

Because there is no evidence of physical joint damage in close to $75 \%$ of individuals with knee pain, it is reasonable to assume that joint misuse plays a significant role in the etiology of the reported pain. Correspondingly, the ability to identify knee muscle misuse patterns which can be readily corrected through exercise or behavioral changes could provide a simple and effective means to ameliorate knee pain and preclude progressive damage that would eventually result in corrective surgery and/or eventual knee replacement. This study provides confirmation that VMG analysis may be capable of providing this capability in the clinic.

\section{ACKNOWLEDGEMENTS}

This study was supported in part by the Clinical Science and Engineering Research Center at Binghamton University, the Southern Tier Center on Aging, and the Center for Advanced Sensor Technology at Stony Brook University. We would like to thank Swapan Mookerjee, Matt McMahon, Jason Cole, Sree Koneru, Amy Chaffee, Tim Cortesi, and Chuck Schwerin for their assistance in the manuscript preparation.

\section{REFERENCES}

[1] National Center for Health Statistics. Health (2011) United States, 2010: With special feature on death and dying. Hyattsville, MS.

[2] Jinks, C., Jordan, K. and Croft, P. (2002) Measuring the population impact of knee pain and disability with the Western Ontario and McMaster Universities Osteoarthritis Index (WOMAC). Pain, 100, 55-64. doi:10.1016/S0304-3959(02)00239-7

[3] Nguyen, U.D.T., Zhang, Y., Zhu, Y., Niu, J., Zhang, B. and Felson, D.T. (2011) Increasing prevalence of knee pain and symptomatic knee osteoarthritis: Survey and cohort data. Annals of Internal Medicine, 155, 725-732.

[4] Muraki, S., Dennison, E., Jameson, K., Boucher, B.J., Akune, T., Yoshimura, N., Judge, A., Arden, N.K., Javaid, K. and Cooper, C. (2011) Association of vitamin D status with knee pain and radiographic knee osteoarthritis. Os- 
teoarthritis and Cartilage, 19, 1301-1306. doi:10.1016/j.joca.2011.07.017

[5] Hurley, M.V. (1999) The role of muscle weakness in the pathogenesis of osteoarthritis. Rheumatic Disease Clinics of North America, 25, 283-298. doi:10.1016/S0889-857X(05)70068-5

[6] Becker, R., Berth, A., Mehring, M. and Awlszus. F. (2004) Neuromuscular quadriceps dysfunction prior to osteoarthritis of the knee. Journal of Orthopaedic Research, 22, 768-773. doi:10.1016/j.orthres.2003.11.004

[7] Bennell, K.L. and Hinman, R.S. (2011) A review of the clinical evidence for exercise in osteoarthritis of the hip and knee. Journal of Science and Medicine in Sport, 14, 4-9. doi:10.1016/j.jsams.2010.08.002

[8] Hochberg, M.C., Altman, R.D., April, K.T., Benkhalti, M., Guyatt, G., McGowan, J., Towheed, T., Welch, V., Wells, G. and Tugwell, P. (2012) American college of rheumatology 2012 recommendations for the use of non-pharmacologic and pharmacologic therapies in osteoarthritis of the hand, hip and knee. Arthritis Care \& Research, 9 January 2012 (epub ahead of print).

[9] Cerny, K. (1995) Vastus medialis oblique/vastus lateralis muscle activity ratios for selected exercises in persons with and without patellofemoral pain syndrome. Physical Therapy, 75, 672-682.

[10] Cowan, S.M., Binnell, K.L., Hodges, P.W., Crossley, K.M. and McConnel, J.M. (2001) Delayed onset of electromyographic activity of vastus medialis obliquus relative to vastus lateralis in subjects with patellofemoral pain syndrome. Archives of Physical Medicine and Rehabilitation, 82, 183-189. doi:10.1053/apmr.2001.19022

[11] Hurley, M.V. (2003) Muscle dysfunction and effective rehabilitation of knee osteoarthritis: What we know and what we need to find out. Arthritis \& Rheumatism, 49, 444-452. doi:10.1002/art.11053

[12] Hortobagyi, T., Westerkamp, L., Beam, S., Moody, J., Garry, J., Holbert, D. and De Vita, P. (2004) Altered ham-string-quadriceps muscle balance in patients with knee osteoarthritis. Clinical Biomechanics, 20, 97-104. doi:10.1016/j.clinbiomech.2004.08.004

[13] Chang, A., Hayes, K., Dunlop, D., Song, J., Hurwitz, D., Cahue, S. and Sharma, L. (2005) Hip abduction moment and protection against medial tibiofemoral osteoarthritis progression. Arthritis \& Rheumatism, 52, 3515-3519. doi:10.1002/art.21406

[14] Sledm, E.A., Khoja, L., Deluzio, K.J., Olney, S.J. and Culham, E.G. (2010) Effect of a home program of hip abductor exercises on knee joint loading, strength, function, and pain in people with knee osteoarthritis: A clini- cal trial. Physical Therapy, 90, 895-904. doi: $10.2522 / \mathrm{ptj} .20090294$

[15] Matheson, G.O., Maffey-Ward, L., Mooney, M., Ladly, K., Fung, T. and Zhang, Y.-T. (1997) Vibromyography as a quantitative measure of muscle force production. Scandinavian Journal of Rehabilitation Medicine, 29, 29-35.

[16] Madeleine, P., Bajaj, P., Sogaard, K. and Arendt-Nielsen, L. (2001) Mechanomyography and electromyography force relationships during concentric, isometric and eccentric contractions. Journal of Electromyography and Kinesiology, 11, 113-121. doi:10.1016/S1050-6411(00)00044-4

[17] Orizio, C., Gobbo, M., Diemont, B., Esposito, F. and Veicsteinas, A. (2003) The surface mechanomyogram as a tool to describe the influence of fatigue on biceps brachii motor unit activation strategy. Historical basis and novel evidence. European Journal of Applied Physiology, 90, 326-336. doi:10.1007/s00421-003-0924-1

[18] Tian, S.-L., Liu, Y., Li, L., Fu, W.-J. and Peng, C.-H. (2010) Mechanomyography is more sensitive than EMG in detecting age-related sarcopenia. Journal of Biomechanics, 43, 551-556.

doi:10.1016/j.jbiomech.2009.09.034

[19] Archer, A.A., Atangcho. P., Sabra, K.G. and Shinohara, M. (2011) Propagation direction of natural mechanical oscillations in the biceps brachii muscle during voluntary contraction. Journal of Electromyography Kinesiology, 22, 51-59. doi:10.1016/i.jelekin.2011.09.011

[20] Cole, J., Madhavan, G. and McLeod, K.J. (2006) Vibromyographic quantification of voluntary isometric contractile force in the brachioradialis. IEEE Engineering in Medicine and Biology Society, 1, 1708-1710.

[21] Beck, T.W., Housh, T.J., Fry, A.C., Cramer, J.T., Weir, J.P., Schilling, B.K., Falvo, M.J. and Moore, C.A. (2009) Wavelet-based analysis of surface mechanomyographic signals from subjects with differences in myosin heavy chain isoform content. Electromyography and Clinical Neurophysiology, 49, 167-175.

[22] McLeod, K.J. and Cole, J. (2011) Realtime assessment of muscle effort during open and closed chain activities. USPTO Pub. No. 2011/0196262A1.

[23] Grood, E.S., Suntay, W.J., Noyes, F.R. and Butler, D.L. (1984) Biomechanics of the knee extension exercise. Journal of Bone Joint Surgery, 66A, 725-734.

[24] Jonssonm P. and Alfredson, H. (2005) Superior results with eccentric compared to concentric quadriceps training in patients with jumper's knee: A prospective randomized study. Journal of Sports Medicine, 9, 847-850. 